\title{
BMJ Open Sleep, biological rhythms and anxiety in the perinatal period: a systematic review protocol
}

\author{
Jasmyn E A Cunningham (1) ," Aljeena R Qureshi (D) ," Sheryl M Green (D) ,3,4 \\ Benicio N Frey (D) , , ${ }^{3,5}$ Anastasiya Slyepchenko (i) ${ }^{3}$
}

To cite: Cunningham JEA, Qureshi AR, Green SM, et al. Sleep, biological rhythms and anxiety in the perinatal period: a systematic review protocol. BMJ Open 2021;11:e046767. doi:10.1136/ bmjopen-2020-046767

- Prepublication history and additional supplemental material for this paper are available online. To view these files, please visit the journal online (http://dx.doi.org/10.1136/ bmjopen-2020-046767).

Received 09 November 2020 Accepted 22 July 2021

Check for updates

(c) Author(s) (or their employer(s)) 2021. Re-use permitted under CC BY-NC. No commercial re-use. See rights and permissions. Published by BMJ.

${ }^{1}$ Michael G DeGroote School of Medicine, McMaster University, Hamilton, Ontario, Canada ${ }^{2}$ Health Sciences Honours Program, McMaster University, Hamilton, Ontario, Canada

${ }^{3}$ Department of Psychiatry and Behavioural Neurosciences, McMaster University, Hamilton, Ontario, Canada

${ }^{4}$ Women's Health Concerns Clinic, St Joseph's Healthcare Hamilton, Hamilton, Ontario, Canada

${ }^{5}$ Mood Disorders Program, St Joseph's Healthcare Hamilton, Hamilton, Ontario, Canada

Correspondence to Jasmyn E A Cunningham; cunninje@mcmaster.ca

\section{ABSTRACT}

Introduction Pregnancy and new parenthood is an exciting time, but also a stressful life event that can predispose to mental health challenges. Perinatal anxiety is one such challenge, and is an important contributor to parental distress and other negative outcomes. Sleep and biological rhythms are often disrupted in the perinatal period. These disruptions have been associated with postpartum depression, and in some cases with perinatal anxiety. However, the literature concerning the association with perinatal anxiety is inconsistent and may be methodologically limited. To our knowledge, there has been no comprehensive review published characterising the relationships between sleep, biological rhythms, and perinatal anxiety and related disorders to date. In this systematic review, we will summarise the current state of the literature concerning these relationships, allowing us to highlight gaps and potentially inform clinical understanding of perinatal anxiety, sleep and biological rhythms.

Methods and analysis Primary research articles will be eligible for inclusion if they assess perinatal anxiety or related disorders using validated criteria (self-report or diagnostic), assess sleep and biological rhythms in the perinatal period, include $>4$ participants and meet other inclusion/exclusion criteria. We will conduct comprehensive searches of MEDLINE, PsycINF0, Embase and CINAHL, with coverage spanning from database conception to search date (August 1, 2021). Key search concepts include (1) the perinatal period, (2) sleep/biological rhythms and (3) anxiety. Risk of bias will be evaluated using the Cochrane Risk of Bias Tool. Data will be narratively synthesised, with quantitative synthesis included if possible. When relevant, strength of evidence will be assessed using Grading of Recommendations Assessment, Development and Evaluation criteria, and potential publication bias will be assessed.

Ethics and dissemination Research ethics approval is not required. Study results will be reported according to Preferred Reporting Items for Systematic Reviews and MetaAnalyses guidelines. Results will be disseminated to relevant stakeholders as conference presentation(s) and submitted for publication in a peer-reviewed journal.

PROSPERO registration number 200166 .

\section{INTRODUCTION}

\section{Rationale}

While pregnancy and new parenthood is often regarded as an exciting and positive time, it is also a stressful life event, ${ }^{1}$ with many

\section{Strengths and limitations of this study}

- Broad search and inclusion criteria will allow for a comprehensive review characterising the relationships between sleep, biological rhythms, and perinatal anxiety and related disorders, which will highlight gaps in current understanding of these relationships.

- Given the state of the literature, the authors predict few included studies per research question and a large degree of heterogeneity between them (eg, in methods of assessment of anxiety, sleep and biological rhythms), which will limit the ability for quantitative analysis.

- In this study, we have defined 'perinatal' as pregnancy and up to 12 months postpartum, as opposed to several weeks postpartum, as many of these individuals will continue to experience disruptions and mental health challenges for this more extended period.

accompanying changes and disruptions that can predispose new parents and new parentsto-be to mental health challenges during pregnancy and the postpartum (the perinatal period). Depression is the most studied mental disorder occurring in this period, affecting as many as $19 \%$ of persons during the first 3 months postpartum, ${ }^{2}$ and potentially contributing to negative outcomes not only for the postpartum individual but also for their family. ${ }^{3}$ However, despite receiving less research attention, perinatal anxiety is also an important contributor to parental distress, ${ }^{4}$ and often co-occurs with depression, making both disorders more difficult to treat. ${ }^{4-7}$ Given its common co-occurrence with depression, anxiety is often studied in the context of this disorder; however, it is important to distinguish the differential impact of anxiety from depression given its respective contribution to distress as well as to better inform appropriate treatment options. ${ }^{4}$ While not formally acknowledged as a disorder in the Diagnostic and Statistical Manual of Mental Disorders, 
Fifth Edition (DSM-5), ${ }^{8}$ postpartum anxiety has a prevalence of approximately $15 \%-18 \%$ (self-reported symptoms) and 9\%-10\% (diagnosed) in developed countries, ${ }^{6}$ and is associated with negative outcomes. ${ }^{910}$ Similarly, the prevalence of anxiety symptoms during pregnancy ranges from $18 \%$ to $24 \%$ (self-reported) and $15 \%$ to $18 \%$ (clinical diagnosis). ${ }^{6}$

Accumulating evidence suggests that perinatal anxiety may negatively impact outcomes in peripartum individuals and their infants. In peripartum individuals, perinatal anxiety may affect not only psychosocial outcomes, such as parental self-confidence and body image, but also obstetric outcomes, such as sick leave during pregnancy, increased visits to obstetricians, anaemia, delivery through C-sections and delivery prior to term. ${ }^{11-13}$ Importantly, anxiety disorders during the perinatal period also appear to negatively influence infant outcomes, including birth weight, Apgar scores, parenting behaviour, the relationship between mothers and their infants (including bonding - a key process in the development of a child), and excessive crying in infants. ${ }^{11} 14-17$ Emerging evidence suggests that perinatal anxiety disorders are predictive of children's emotional and behavioural disturbances at 4 years of age. ${ }^{18}$ Thus, prevention and treatment of perinatal anxiety can potentially have a positive impact on both the parent and their child.

Although no longer formally categorised as anxiety disorders in the DSM-5, anxiety-related disorders such as obsessive-compulsive disorder (OCD) and post-traumatic stress disorder (PTSD) are also prevalent in the perinatal period and have been associated with poor health outcomes for mothers and infants. The existing literature shows that the perinatal period represents a time of increased risk for the development and exacerbation of OCD symptoms, with an estimated prevalence of $0.2 \%-3.5 \%$ in pregnancy and up to $2.3 \%-9 \%$ in the postpartum. ${ }^{19-21}$ OCD during pregnancy is frequently associated with contamination fears regarding the fetus, and can present with compulsions such as excessive washing or cleaning rituals, diet restriction and restriction of contact with others postnatally. Postnatal onset of OCD has been associated with aggressive intrusion cognitions involving deliberately harming the baby and is associated with the avoidance of caregiving tasks such as bathing, or compulsive checking, for example that the infant has not been kidnapped by a stranger. ${ }^{19}{ }^{20}$ While literature regarding PTSD during the perinatal period is relatively limited both in quantity and in methodological quality, PTSD during the postpartum period has also been associated with negative outcomes including low birth weight and decreased rates of breast feeding. ${ }^{22}$

Sleep and biological rhythms are also commonly disrupted in the perinatal period. ${ }^{23-26}$ In the general population, disturbed sleep has been associated with diabetes, obesity, metabolic syndrome, chronic pain, hypertension and heart disease, ${ }^{27-29}$ and sleep deprivation can contribute to changes in immune and endocrine functioning, as well as cognition and aspects of attention. ${ }^{30-33}$ Poor sleep and the presence of sleep disorders are also associated with a lower quality of life, and interference with performance of important activities such as driving. ${ }^{34}$ Specifically, during pregnancy, poor quality of sleep is associated with negative outcomes such as increased risk of suicidal ideation, preterm birth, low birth weight, fetal growth restriction and gestational diabetes. $^{36-38}$

Sleep disruption has also been associated with postpartum depression, ${ }^{26} 39$ and in some cases with perinatal anxiety. ${ }^{3940}$ However, the literature concerning the association with perinatal anxiety and related disorders is inconsistent. For example, in one study, women who reported poor sleep had higher self-reported anxiety symptoms at 6 months postpartum. ${ }^{39}$ Similarly, other studies have reported that anxiety was associated with subjective, but not objective, sleep changes in the third trimester, ${ }^{41}$ and higher levels of anxiety and symptoms of OCD were reported among individuals experiencing mid-pregnancy insomnia. $^{21}$ In contrast, Tham and colleagues showed that postnatal depression, but not anxiety, is associated with poor sleep quality during pregnancy. ${ }^{42}$ Sleep difficulties are also common in PTSD,${ }^{43}$ and sleep difficulties in the perinatal period have been associated with a worsening trajectory of post-traumatic stress across this same period. ${ }^{44}$ Additionally, fewer studies have focused on the relationships between perinatal anxiety and biological rhythms other than sleep, although perinatal alterations in biological rhythms have been linked to negative mood outcomes. $^{252645}$ Published literature concerning sleep, biological rhythms, and perinatal anxiety and related disorders in many cases may also be limited by small sample sizes, heterogeneity and other methodological shortcomings. ${ }^{26}$ Despite the negative outcomes associated with both anxiety and disturbed sleep during the perinatal period, ${ }^{491036-38}$ to our knowledge, there has been no comprehensive review published characterising the relationships between these concepts to date. In this systematic review, we will summarise the current state of the literature concerning the relationships between sleep, biological rhythms, and perinatal anxiety and related disorders, which will allow us to highlight gaps in the current understanding of these phenomena, as well as potentially inform clinical understanding of these constructs.

\section{Objectives}

Our aim is to elucidate the interactions between sleep, biological rhythms, and anxiety and related disorders in the perinatal period (including pregnancy and up to 12 months postpartum). To do so, this systematic review will address the following research questions:

1. How do sleep and biological rhythms in the perinatal period affect the onset and course of perinatal anxiety and related disorders, and vice versa?

2. How do sleep and biological rhythms in the perinatal period affect the outcomes of perinatal anxiety and related disorders? 
3. How do perinatal anxiety and related disorders affect outcomes of sleep and biological rhythm disruptions?

4. Are there any chronotherapeutic treatments that have been administered in the perinatal period to treat anxiety and related disorders, or sleep disturbances in individuals with anxiety and related disorders?

5 . What are the effects of treatment for perinatal anxiety and related disorders on sleep and circadian rhythms? Are there any treatments for perinatal anxiety and related disorders targeting sleep or biological rhythms that have been implemented? What were the effects of the implemented treatments?

\section{METHODS AND ANALYSIS}

This protocol and the reporting of results will be based on Preferred Reporting Items for Systematic Reviews and Meta-Analyses guidelines. ${ }^{46}$

\section{Eligibility criteria}

Studies will be eligible for inclusion if they meet the following criteria: (a) assess perinatal anxiety using a validated self-report tool or diagnostic criteria (including anxiety symptoms or disorders; or PTSD, OCD or their symptoms); (b) assess sleep or biological rhythms in the perinatal period, either subjectively or objectively (including clinical questionnaires, clinical interviews, actigraphy, polysomnography, etc); (c) published in or translated to English; and (d) primary research article with $>4$ human participants. Studies will be excluded if they: (a) include jet lag as their sole sleep disturbance; (b) include duplicate data from a database or other included studies; (c) do not distinguish adequately between perinatal anxiety and symptoms of other mental disorders (such as depression).

\section{Information sources and search strategy}

Comprehensive searches of MEDLINE, PsycINFO, Embase and CINAHL will be conducted to identify relevant articles for inclusion. Coverage spans from 1946 (MEDLINE), 1806 (PsycINFO), 1974 (Embase) and 1981 (CINAHL) to search date (August 1, 2021). The search strategy will include predetermined keywords and subject headings appropriate to each database (Medical Subject Headings for MEDLINE, etc). See online supplemental appendix 1 for terms and sample search strategy. Key concepts include (1) the perinatal period (pregnancy and/or up to 12 months postpartum), (2) sleep and/or biological rhythms, and (3) anxiety. No search limits will be applied.

\section{Screening and data extraction}

Citation lists from searches will be imported to EndNote V.20 for de-duplication. Following de-duplication, citations and data will be managed using Covidence. Titles and abstracts will be independently screened by two reviewers for potential relevance. Following this, full text of potentially relevant articles will be obtained and independently assessed for inclusion. In cases of disagreement at either stage, a third reviewer will assess the article for potential inclusion. Two reviewers will independently extract data using a standardised, electronic data collection form embedded within Covidence. Data will be reviewed for clarity and consistency by a third reviewer, and reconciled into one document.

Data will be extracted for the following variables: (1) assessment/diagnosis of sleep and/or biological rhythms and associated disturbances, and related information such as prevalence, course, treatment outcomes, etc; (2) assessment/diagnosis of anxiety or related disorder, and related information such as prevalence, course, treatment outcomes, etc; (3) characteristics of included participants, including pregnancy-related and childbirthrelated information, as well as the presence of spousal/ partner/familial support persons; (4) study details, such as year of publication, number of included participants, comparator groups, methods of recruitment, timing and type of assessments, cut-off points, statistical analysis methods and main findings. The first two domains of variables related to sleep, biological rhythms and anxiety are the main outcomes of interest. It should be noted that methods of measuring sleep and biological rhythms as well as methods of assessment of anxiety and related disorders will likely vary substantially between the studies. Current methods of assessment of sleep and biological rhythms are varied and may include subjective questionnaires (eg, the Pittsburgh Sleep Quality Index, ${ }^{47}$ the Insomnia Severity Index ${ }^{48}$ the Biological Rhythms Interview of Assessment in Neuropsychiatry ${ }^{49}$ ), actigraphy, polysomnography, measurement of body temperature and fluctuation in hormones such as melatonin among others. Though there is no perinatal-specific anxiety diagnosis included in the DSM-5, a diagnosis of an anxiety disorder may be established using validated clinical interviews (eg, the Mini International Neuropsychiatric Interview, the Structured Clinical Interview for the DSM-5), and clinical questionnaires may be used to assess symptom severity (eg, the State-Trait Anxiety Inventory, the Generalized Anxiety Disorder-7, the Yale-Brown Obsessive Compulsive Scale, and the Depression, Anxiety and Stress Scale). Some perinatal-specific anxiety scales exist, including the Perinatal Obsessive-Compulsive Scale, the anxiety subscale of the Edinburgh Postnatal Depression Scale and the Perinatal Anxiety Screening Scale.

\section{Data synthesis and evaluation}

Risk of bias will be evaluated using the Cochrane Risk of Bias Tool. ${ }^{50}$ Risk of bias will be assessed at the level of the study. Study data will be narratively synthesised. Quantitative synthesis will be included if possible, however given the state of the literature on the topic, the authors predict a large degree of study heterogeneity and a low number of relevant studies per research question, which may limit the appropriateness of quantitative methods. Metaregression, subgroup analysis, and/or random effects meta-analysis may be employed if appropriate given the high degree of expected heterogeneity. When relevant, 
strength of evidence will be assessed using Grading of Recommendations Assessment, Development and Evaluation criteria, and potential publication bias will be assessed.

\section{Patient and public involvement}

This study protocol was presented to the Community Advisory Committee consisting of former patients of the Women's Health Concerns Clinic at St Joseph's Healthcare Hamilton (March 2021, Hamilton, Ontario, Canada). Two individuals with lived experience have read and provided feedback on this systematic review protocol.

\section{ETHICS AND DISSEMINATION}

Research ethics approval is not required. Results will be disseminated to relevant stakeholders as conference presentation(s) and submitted for publication in a peerreviewed journal.

\section{DISCUSSION}

Understanding the complex relationships of perinatal anxiety with sleep and biological rhythms will allow researchers and clinicians to begin to assess the importance of perinatal sleep and biological rhythms as risk factors and aetiological components of anxiety during this period. As part of this review, we aim to provide a comprehensive and detailed overview of the existing literature on this topic, and to provide a foundation for future studies aiming to fill the gaps in current understanding of these relationships. Notably, a previous systematic review identified comorbid sleep disorders to be a risk factor for the first-time onset of anxiety during the perinatal period, ${ }^{51}$ indicating an important role of sleep in the development of anxiety during this time. If a substantial relationship is identified among sleep, biological rhythms and anxiety during the perinatal period, it is possible that sleep and biological rhythms may offer targets for pharmacological or psychotherapeutic treatment and prevention of anxiety during this critical period in the lives of parents, parents-to-be and their families.

\section{Strengths and limitations}

Given the state of the current literature, the authors predict that few studies will address each of the research questions included in this study. Additionally, a large degree of heterogeneity is expected in the assessment of sleep and biological rhythms and of perinatal anxiety in the literature, due to the diverse range of methods currently used to assess these outcomes (eg, actigraphy, polysomnography, subjective questionnaires, clinical interviews). This may limit our ability to conduct a quantitative analysis.

However, a strength of our protocol lies in including a broad search and inclusion criteria which will allow us to comprehensively describe these phenomena, and to highlight the current literature describing their inter-relationships. Another strength of our protocol is the definition of 'perinatal' as including pregnancy and up to 12 months postpartum, as opposed to merely pregnancy and several weeks immediately following childbirth. Many individuals continue to experience disruptions in their mental health related to perinatal-specific stressors in this extended period, and thus a more comprehensive characterisation has the potential to benefit these individuals throughout longer duration following childbirth.

Acknowledgements We would like to extend our gratitude to Christine Vanderveen and Brydie Huffman for providing feedback on the study protocol.

Contributors JEAC contributed to study design, drafted and critically revised the research protocol. ARQ contributed to study design, drafted and critically revised the research protocol. SMG critically revised the research protocol. BNF contributed to study idea and critical revision of the research protocol, and provided research supervision. AS conceived of the study idea; contributed to study design and critical revision of the research protocol; and provided research supervision.

Funding This work is sponsored in part by the Michael G DeGroote School of Medicine McMaster Medical Student Research Excellence Award (MAC RES) and by an unrestricted fundraising gift from Shoppers Drug Mart (Shoppers Run For Women).

Competing interests AS has received research funding from Pfizer.

Patient consent for publication Not required.

Provenance and peer review Not commissioned; externally peer reviewed.

Supplemental material This content has been supplied by the author(s). It has not been vetted by BMJ Publishing Group Limited (BMJ) and may not have been peer-reviewed. Any opinions or recommendations discussed are solely those of the author(s) and are not endorsed by BMJ. BMJ disclaims all liability and responsibility arising from any reliance placed on the content. Where the content includes any translated material, BMJ does not warrant the accuracy and reliability of the translations (including but not limited to local regulations, clinical guidelines, terminology, drug names and drug dosages), and is not responsible for any error and/or omissions arising from translation and adaptation or otherwise.

Open access This is an open access article distributed in accordance with the Creative Commons Attribution Non Commercial (CC BY-NC 4.0) license, which permits others to distribute, remix, adapt, build upon this work non-commercially, and license their derivative works on different terms, provided the original work is properly cited, appropriate credit is given, any changes made indicated, and the use is non-commercial. See: http://creativecommons.org/licenses/by-nc/4.0/.

\section{ORCID iDs}

Jasmyn E A Cunningham http://orcid.org/0000-0002-7067-8832

Aljeena R Qureshi http://orcid.org/0000-0001-9462-6482

Sheryl M Green http://orcid.org/0000-0002-2226-8003

Benicio N Frey http://orcid.org/0000-0001-8267-943X

Anastasiya Slyepchenko http://orcid.org/0000-0003-0179-2129

\section{REFERENCES}

1 Holmes TH, Rahe RH. The social Readjustment rating scale. $J$ Psychosom Res 1967;11:213-8.

2 Gavin NI, Gaynes BN, Lohr KN, et al. Perinatal depression: a systematic review of prevalence and incidence. Obstet Gynecol 2005;106:1071-83.

3 Weissman MM. Postpartum depression and its long-term impact on children: many new questions. JAMA Psychiatry 2018;75:227-8.

4 Miller RL, Pallant JF, Negri LM. Anxiety and stress in the postpartum: is there more to postnatal distress than depression? BMC Psychiatry 2006;6:12.

5 Falah-Hassani K, Shiri R, Dennis C-L. Prevalence and risk factors for comorbid postpartum depressive symptomatology and anxiety. $J$ Affect Disord 2016;198:142-7.

6 Dennis C-L, Falah-Hassani K, Shiri R. Prevalence of antenatal and postnatal anxiety: systematic review and meta-analysis. $\mathrm{Br} \mathrm{J}$ Psychiatry 2017;210:315-23.

7 Hawke LD, Relihan J, Miller J, et al. Engaging youth in research planning, design and execution: practical recommendations for researchers. Health Expect 2018;21:944-9. 
8 American Psychiatric Association. Diagnostic and statistical manual of mental disorders. 5 edn. Arlington, VA: American Psychiatric Publishing, 2013.

9 Glover V. Maternal depression, anxiety and stress during pregnancy and child outcome; what needs to be done. Best Pract Res Clin Obstet Gynaecol 2014;28:25-35.

10 Stein A, Pearson RM, Goodman SH, et al. Effects of perinatal mental disorders on the fetus and child. Lancet 2014;384:1800-19.

11 Goodman JH, Watson GR, Stubbs B. Anxiety disorders in postpartum women: a systematic review and meta-analysis. J Affect Disord 2016;203:292-331.

12 Andersson L, Sundström-Poromaa I, Wulff M, et al. Implications of antenatal depression and anxiety for obstetric outcome. Obstet Gynecol 2004; 104:467-76.

13 Bánhidy F, Acs N, Puhó E, et al. Association between maternal panic disorders and pregnancy complications and delivery outcomes. Eur $J$ Obstet Gynecol Reprod Biol 2006;124:47-52.

14 Petzoldt J, Wittchen H-U, Wittich J, et al. Maternal anxiety disorders predict excessive infant crying: a prospective longitudinal study. Arch Dis Child 2014;99:800-6.

15 Murray L, Cooper P, Creswell C, et al. The effects of maternal social phobia on mother-infant interactions and infant social responsiveness. J Child Psychol Psychiatry 2007:48:45-52.

16 Berle J Ø, Mykletun A, Daltveit AK, et al. Neonatal outcomes in offspring of women with anxiety and depression during pregnancy. A linkage study from the Nord-Trøndelag health study (Hunt) and medical birth registry of Norway. Arch Womens Ment Health 2005;8:181-9.

17 Grigoriadis S, Graves L, Peer M, et al. Maternal anxiety during pregnancy and the association with adverse perinatal outcomes: systematic review and meta-analysis. J Clin Psychiatry 2018:79. doi:10.4088/JCP.17r12011. [Epub ahead of print: 04 Sep 2018].

18 O'Connor TG, Heron J, Golding J, et al. Maternal antenatal anxiety and children's behavioural/emotional problems at 4 years. Report from the Avon longitudinal study of parents and children. $\mathrm{Br} \mathrm{J}$ Psychiatry 2002;180:502-8.

19 Frías Álvaro, Palma C, Barón F, et al. Obsessive-compulsive disorder in the perinatal period: Epidemiology, phenomenology, pathogenesis, and treatment. [Trastorno obsesivo-compulsivo durante el período perinatal: epidemiología, fenomenología, etiopatogenia y tratamiento]. Anales de Psicología 2015;31.

20 Challacombe FL, Wroe AL. A hidden problem: consequences of the misdiagnosis of perinatal obsessive-compulsive disorder. $\mathrm{Br} J \mathrm{Gen}$ Pract 2013;63:275-6.

21 Osnes RS, Eberhard-Gran M, Follestad T, et al. Mid-pregnancy insomnia is associated with concurrent and postpartum maternal anxiety and obsessive-compulsive symptoms: a prospective cohort study. J Affect Disord 2020;266:319-26.

22 Cook N, Ayers S, Horsch A. Maternal posttraumatic stress disorder during the perinatal period and child outcomes: a systematic review. $J$ Affect Disord 2018;225:18-31.

23 Facco FL, Kramer J, Ho KH, et al. Sleep disturbances in pregnancy. Obstet Gynecol 2010;115:77-83.

24 Signal TL, Gander PH, Sangalli MR, et al. Sleep duration and quality in healthy nulliparous and multiparous women across pregnancy and post-partum. Aust N Z J Obstet Gynaecol 2007;47:16-22.

25 Sharkey KM, Pearlstein TB, Carskadon MA. Circadian phase shifts and mood across the perinatal period in women with a history of major depressive disorder: a preliminary communication. $J$ Affect Disord 2013;150:1103-8.

26 Gallaher KGH, Slyepchenko A, Frey BN, et al. The role of circadian rhythms in postpartum sleep and mood. Sleep Med Clin 2018;13:359-74.

27 Foley D, Ancoli-Israel S, Britz P, et al. Sleep disturbances and chronic disease in older adults: results of the 2003 national sleep Foundation sleep in America survey. J Psychosom Res 2004;56:497-502.

28 Hall MH, Muldoon MF, Jennings JR, et al. Self-reported sleep duration is associated with the metabolic syndrome in midlife adults. Sleep 2008;31:635-43.
29 Gottlieb DJ, Redline S, Nieto FJ, et al. Association of usual sleep duration with hypertension: the sleep heart health study. Sleep 2006;29:1009-14.

30 Cunningham JEA, Jones SAH, Eskes GA, et al. Acute sleep restriction has differential effects on components of attention. Front Psychiatry 2018;9:499.

31 Van Dongen HPA, Maislin G, Mullington JM, et al. The cumulative cost of additional wakefulness: dose-response effects on neurobehavioral functions and sleep physiology from chronic sleep restriction and total sleep deprivation. Sleep 2003;26:117-26.

32 Spiegel K, Leproult R, Van Cauter E. Impact of sleep debt on metabolic and endocrine function. Lancet 1999;354:1435-9.

33 Spiegel K, Sheridan JF, Van Cauter E. Effect of sleep deprivation on response to immunization. JAMA 2002;288:1471-2.

34 Reimer MA, Flemons WW. Quality of life in sleep disorders. Sleep Med Rev 2003;7:335-49.

35 Fairclough SH, Graham R. Impairment of driving performance caused by sleep deprivation or alcohol: a comparative study. Hum Factors 1999;41:118-28.

36 Cai S, Tan S, Gluckman PD, et al. Sleep quality and nocturnal sleep duration in pregnancy and risk of gestational diabetes mellitus. Sleep 2017;40. doi:10.1093/sleep/zsw058. [Epub ahead of print: 01 Feb 2017].

37 Gelaye B, Barrios YV, Zhong Q-Y, et al. Association of poor subjective sleep quality with suicidal ideation among pregnant Peruvian women. Gen Hosp Psychiatry 2015;37:441-7.

38 Micheli K, Komninos I, Bagkeris E, et al. Sleep patterns in late pregnancy and risk of preterm birth and fetal growth restriction. Epidemiology 2011;22:738-44.

39 Okun ML, Mancuso RA, Hobel CJ, et al. Poor sleep quality increases symptoms of depression and anxiety in postpartum women. J Behav Med 2018;41:703-10.

40 Lawson A, Murphy KE, Sloan E, et al. The relationship between sleep and postpartum mental disorders: a systematic review. J Affect Disord 2015;176:65-77.

41 Bei B, Milgrom J, Ericksen J, et al. Subjective perception of sleep, but not its objective quality, is associated with immediate postpartum mood disturbances in healthy women. Sleep 2010;33:531-8.

42 Tham EKH, Tan J, Chong Y-S, et al. Associations between poor subjective prenatal sleep quality and postnatal depression and anxiety symptoms. J Affect Disord 2016;202:91-4.

43 Vasdev S, Cunningham J, Shapiro C. Sleep changes in PTSD, in sleep and combat-related post traumatic stress disorder. New York, NY: Springer Science+Business Media LLC, 2018: 201-8.

44 Paulson JL, Miller-Graff L. Prenatal sleep quality and mental health symptoms across the perinatal period: a longitudinal study of highrisk women. J Psychosom Res 2019;116:31-6.

45 Krawczak EM, Minuzzi L, Hidalgo MP, et al. Do changes in subjective sleep and biological rhythms predict worsening in postpartum depressive symptoms? A prospective study across the perinatal period. Arch Womens Ment Health 2016;19:591-8.

46 Moher D, Liberati A, Tetzlaff J, et al. Preferred reporting items for systematic reviews and meta-analyses: the PRISMA statement. PLoS Med 2009;6:e1000097.

47 Buysse DJ, Reynolds CF, Monk TH, et al. The Pittsburgh sleep quality index: a new instrument for psychiatric practice and research. Psychiatry Res 1989;28:193-213.

48 Bastien $\mathrm{CH}$, Vallières $\mathrm{A}$, Morin $\mathrm{CM}$. Validation of the insomnia severity index as an outcome measure for insomnia research. Sleep Med 2001;2:297-307.

49 Giglio LMF, Magalhães PVdaS, Andreazza AC, et al. Development and use of a biological rhythm interview. J Affect Disord 2009;118:161-5.

50 Higgins JPT, Altman DG, Gøtzsche PC, et al. The Cochrane collaboration's tool for assessing risk of bias in randomised trials. BMJ 2011;343:d5928.

51 Furtado M, Chow CHT, Owais S, et al. Risk factors of new onset anxiety and anxiety exacerbation in the perinatal period: a systematic review and meta-analysis. J Affect Disord 2018;238:626-35. 